Portland State University

PDXScholar

8-1-1995

\title{
Origins and effects of thermal processes on near- field optical probes
}

Andres H. La Rosa

Portland State University

B. I. Yakobson

H. D. Hallen

Follow this and additional works at: https://pdxscholar.library.pdx.edu/phy_fac

Part of the Physics Commons

Let us know how access to this document benefits you.

\section{Citation Details}

La Rosa, A. H., Yakobson, B. I., \& Hallen, H. D. (1995). Origins and effects of thermal processes on nearfield optical probes. Applied Physics Letters, 67(18), 2597.

This Article is brought to you for free and open access. It has been accepted for inclusion in Physics Faculty Publications and Presentations by an authorized administrator of PDXScholar. Please contact us if we can make this document more accessible: pdxscholar@pdx.edu. 


\title{
Origins and effects of thermal processes on near-field optical probes
}

\author{
A. H. La Rosa, B. I. Yakobson, and H. D. Hallen ${ }^{\text {a) }}$ \\ Department of Physics, North Carolina State University, Raleigh, North Carolina 27695
}

(Received 20 July 1995; accepted for publication 22 August 1995)

\begin{abstract}
An aluminum-coated tapered fiber probe, as used in near-field scanning optical microscopy (NSOM), is heated by the light coupled into it. This can destroy the probe or may modify the sample, which can be problematic or used as a tool. To study these thermal effects, we couple modulated visible light of various power through probes. Simultaneously coupled infrared light senses the thermal effects. We report their magnitude, their spatial and temporal scales, and real-time probe damage observations. A model describes the experimental data, the mechanisms for induced IR variation, and their relative importance. (c) 1995 American Institute of Physics.
\end{abstract}

Near-field scanning optical microscopy $(\mathrm{NSOM})^{1}$ extends the application of well-developed conventional (farfield) optics techniques into the mesoscopic world. The high resolution of $\mathrm{NSOM}^{2}$ not possible using conventional optics, is achieved geometrically, by forcing the passage of light through a sub-wavelength-dimension metallic aperture. This aperture is produced at the tip of a tapered optical fiber, and must be scanned in the proximity (near-field region) of the sample under analysis. Measurements are hindered by extremely low light levels coupled through the aperture, e.g., $\sim 10^{-6}$ the input light for a $20 \mathrm{~nm}$ aperture. Most of the light input to the probe is either absorbed or reflected back. Furthermore, the input power is limited by probe heating. Heat may modify the sample or destroy the probe by diffusion of the metal, thus creating a larger, useless, aperture. In this letter, we characterize the thermal effects experimentally and theoretically. Not only do these results characterize potential problems with NSOM application, particularly in dynamic studies, ${ }^{3}$ they also directly impact proximal probe efforts for data storage. ${ }^{4,5}$ In particular, the results identify the model to calculate the maximum rate for writing data.

Figure 1 shows the experimental configuration. Two lasers are input through the tapered fiber probe. An acoustooptical modulator generates visible pulses (50\% duty cycle, 632 or $514 \mathrm{~nm}$ ) used to cause a time-varying temperature near the probe tip. cw infrared $(1.15 \mu \mathrm{m}$ HeNe) light senses the tip condition through its induced modulation at the visible light switching frequency. We have used two NSOM probe geometries characterized by the angles at $\sim 1 \mu \mathrm{m}$ from the end, referred to as slender $\left(20^{\circ}\right)$ and chubby $\left(40^{\circ}\right)$. Both, $\sim 1 \mathrm{~mm}$ long, are fabricated with a commercial puller, ${ }^{6}$ as first modeled in Ref. 7. The aluminum coating thickness varied from 20 to $300 \mathrm{~nm}$ (corrected for rotation and tilt).

The cyclic heating of the probe tip by the visible light effects the passage of IR light in several ways: (a) Thermal expansion of the aperture size " $a$ " results in an increase in the throughput, which scales as $(a / \lambda)^{q} \quad(q=6$ in the Bethe $\operatorname{model}^{8}$ or $q=4$ from a microwave experiment $\left.{ }^{9,10}\right)$. With (Al) thermal expansion coefficient $\kappa=2.4 \times 10^{-5} /{ }^{\circ} \mathrm{C}$ the relative change in IR magnitude is

$$
\Delta \phi / \phi=(1+\kappa \Delta T)^{q}-1 \approx 1.5 \times 10^{-4}\left(\Delta T /{ }^{\circ} \mathrm{C}\right) . \neg
$$

(b) Throughput can decrease due to thermal elongation of the region beyond cutoff (about $\delta z=1 \mu \mathrm{m}$ ) of mode propagation in the cylindrical Al waveguide. Optical intensity can be approximated as exponentially decreasing with attenuation $\alpha \approx 2 \pi / \lambda .{ }^{11}$ A negative variation results, with induced variation: $\Delta \phi / \phi=-\alpha \delta z \kappa \Delta T \approx-10^{-4}\left(\Delta T /{ }^{\circ} \mathrm{C}\right)$. (c) Aluminum is strongly reflecting at IR-visible frequencies, in the relaxation region ${ }^{12}$ where reflectivity is $1-\rho \omega_{p} / 2 \pi$ (plasma frequency $\omega_{p}=2.37 \times 10^{16} \mathrm{~s}^{-1}$ ). With resistivity $\rho=3.1$ $\times 10^{-18} \mathrm{~s}$ and temperature coefficient $4 \times 10^{-3} /{ }^{\circ} \mathrm{C}$, the throughput variation is $\Delta \phi / \phi \approx-0.5 \times 10^{-4}\left(\Delta T /{ }^{\circ} \mathrm{C}\right)$, or more, because more than a single reflection is involved. (d) The optical properties of the fiber dielectric vary with temperature, but these variations are small and similar for the core and cladding, thus producing only a negligible "second order" effect on the throughput. The magnitudes of the three mechanisms (a)-(c) are close, although variations in numerical factors with specific probe geometry can alter their relative weight. Our experimental data suggest the evidence of (a), (b), and (c); the dominant mechanism can vary with the particular probe and as the probe is damaged.

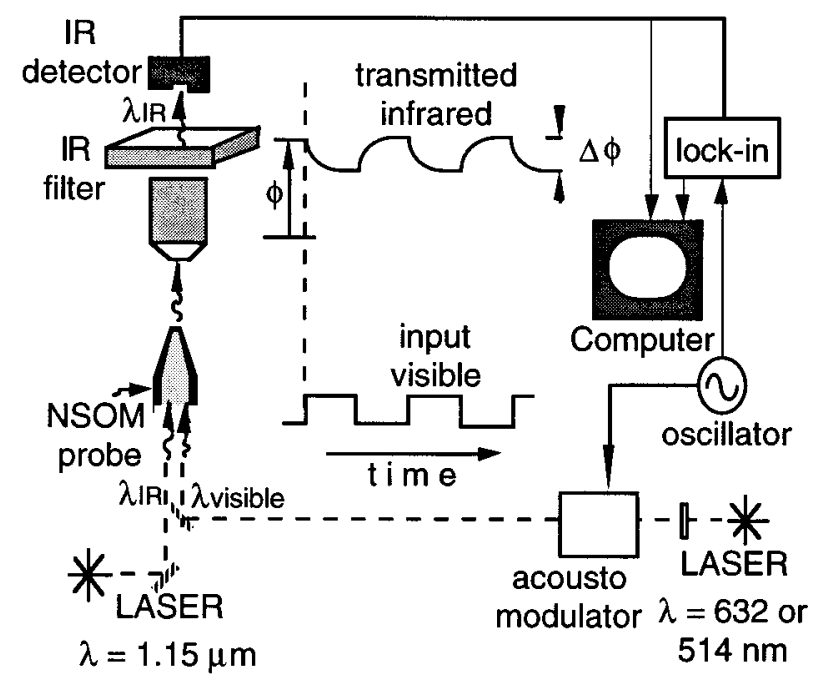

FIG. 1. Schematic of the experiment that extracts information about the thermal properties of the probe. Left-hand side: optical path of the IR radiation. Center: A timing diagram of the process. The visible light modulates the probe temperature which causes a change in the IR transmission. Righthand side: The data is collected either at the frequency of visible light switching or as a base band signal.

${ }^{a)}$ Electronic mail: hans_hallen@ncsu.edu 


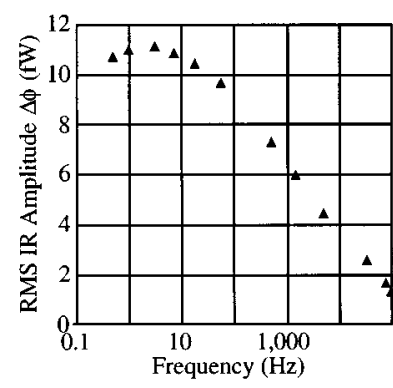

(a)

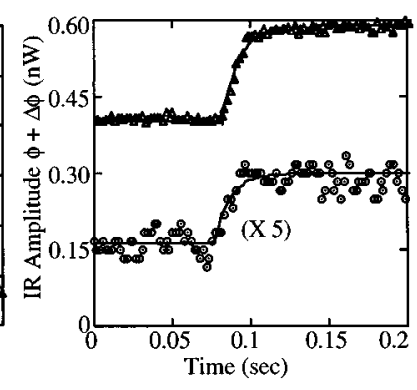

(b)
FIG. 2. The change in IR signal $\Delta \phi$ is measured as a function of (a) frequency (before damage) and (b) time. For both measurements, a slender probe with a $180 \mathrm{~nm}$ thick aluminum coating was used, with $2 \mathrm{~mW}$ red light switching at the frequency shown and detected with $1 \mathrm{~mW}$ of $\mathrm{cw}$ IR input into the probe. The fits shown in (b) yielded values of 0.23 and $0.026 \mathrm{nW}$ pk-pk $\Delta \phi$, and time constants of 10.3 and $10.1 \mathrm{~ms}$ corresponding to frequencies of 15.8 and $15.4 \mathrm{~Hz}$, for the triangles (after damage) and circles (before damage), respectively. The before damage (circle) data have been scaled by a factor of 5 .

The magnitude of the IR signal change can be used to estimate the temperature change near the tip. Experimentally, we find that the normalized IR signal change $(\Delta \phi / \phi)$ grows linearly for small visible light powers (slope $\alpha$ ), then falls below this line at higher powers. A wide range of $|\alpha|$ 's are found for undamaged probes, from 0.015 to $0.15 \mathrm{~mW}^{-1}$. It appears that $|\alpha|$ tends to be smaller $\left(\sim 0.02 \mathrm{~mW}^{-1}\right)$ for thicker Al than $\left(>0.05 \mathrm{~mW}^{-1}\right)$ for thinner $(20 \mathrm{~nm}) \mathrm{Al}$. Preliminary results suggest it may be larger for slender probes. It remains nearly fixed for minor probe damage, but decreases greatly after severe damage. The sign of the correlation with visible light switching (does IR increase or decrease with visible on) helps to identify the mechanism. Both signs are found with undamaged probes, although a negative correlation is more common. When the correlation is positive, the aperture size variation must dominate, and we may use Eq. (1) to estimate the temperature. For a particular case (chubby shape, Al thickness $100 \mathrm{~nm}$ ) with $0.5 \mathrm{~mW}$ peak value of green light and $0.4 \mathrm{~mW}$ of infrared coupled into the fiber, we find $\Delta \phi / \phi=0.008$. This gives $\Delta T=50{ }^{\circ} \mathrm{C}$. When the green input power is raised to $4.5 \mathrm{~mW}$, the probe damages, and $\Delta \phi / \phi=0.058$ or $\Delta T=390{ }^{\circ} \mathrm{C}$.

For thermal time constant $(\tau)$ measurements, it does not matter which mechanism dominates. We have measured $\tau$ in two ways. (1) IR variation is detected with a lock-in amplifier at the frequency of the visible light switching. Data are shown in Fig. 2(a). At low frequencies, the tip temperature and therefore IR signal equilibrate, so the signal is high. At high frequencies, small IR signal variations occur about a steady state. The frequency at which the signal decreases is related to $\tau$. (2) The IR signal is recorded while the visible light is switched [Fig. 2(b)]. The transitions are fit to an exponential to obtain $\tau$. Although the fits yield good agreement, one must be careful when assigning a single $\tau$ to a (generally polychromatic) thermal relaxation. ${ }^{13}$ We find that the $\tau$ 's lie in a narrow range $(8-16 \mathrm{~ms})$ independent of the gross tip shape (slender or chubby), the aluminum coating thickness, and the visible power. It also remains the same as the tip is thermally damaged [Fig. 2(b)]. This suggests that $\tau$ derives from the region which is the same for all probes: the taper far from the aperture.

The thermal time constant can be used to estimate the region of the probe which is heated. Light partially absorbed by the aluminum coating generates heat, which can be dissipated laterally, across the fiber and from the taper back up to the fiber, or longitudinally. Lateral heat transfer through the glass equilibrates its temperature with that of metal coating within the time $\tau_{\perp}=0.4 \times 10^{-6}(\mathrm{r} / \mu \mathrm{m})^{2} \mathrm{~s}$, very short practically everywhere within the taper (current radius $r<60$ $\mu \mathrm{m})$. In contrast, lateral convection or radiation out to the ambient are relatively slow, with the characteristic time $\tau_{c}$ $=0.15(\mathrm{r} / \mu \mathrm{m}) \mathrm{s}$ (several times shorter for radiation at higher temperatures, $T \leqslant 660{ }^{\circ} \mathrm{C}$ ). The probe tip is thus approximated by a cone insulated laterally, so conduction from a hot domain of length $\Delta z$ (measured from the cone apex) upward to the bulk of the fiber resembles the cooling of an entire sphere of radius $\Delta z$. The highly conducting metal coating $\left(k_{\mathrm{al}}=250 k_{\text {glass }}=10^{8} \mu \mathrm{m}^{2} / \mathrm{s}\right)$ enhances the effective thermal diffusivity, which for our probes is large below $\Delta z_{c}=250 \mu \mathrm{m}$ and approaches $\bar{k}=2.5 k_{\text {glass }}$ for $\Delta z>\Delta z_{c}$. Thus, the characteristic time of thermal relaxation is $\tau_{\|}$ $=\Delta z_{c}{ }^{2} / 4 \bar{k}=0.015 \mathrm{~s}$, in good agreement with the measured values. Overall, these estimates indicate that the relaxation time can be associated with thermal conduction from the probe back to the fiber through both glass and metal coating, and that a fairly large part of the taper is warmed above ambient.

The location of maximum energy transfer into the aluminum by imperfect reflections (sideput as opposed to throughput) can be found with a simple experiment. A probe is produced and imaged in Figs. 3(a) and 3(b). Then the probe is severely damaged. Subsequent optical emission from the tip is shown in Fig. 3(c). The bright region $\sim 25 \mu \mathrm{m}$ from the end gives the location. Thermal diffusion has become significant in the heated region and large, discontinuous grains have formed, which allow light to leak. Ray tracing provides additional insight to the sideput location. We use the repeated-zone scheme ${ }^{14}$ to count the number of reflections versus distance within the taper. The resultant sideput density ${ }^{15}$ is shown in Fig. 3(d).

Thermal damage at high visible powers usually results in an increase in the IR signal due to an aperture size increase. Alternatively, a damaged coating can scatter IR light from the probe so that it does not reach the detector, reducing the IR signal. Finally, the IR modulation mechanism, driven by the cyclic heating, may change. Examples are shown in Fig. 4. The data are marked to indicate when the visible light was physically unblocked and blocked. Between, it is switched on and off at $1 \mathrm{~Hz}$ by an acousto-optical modulator. Some data show a jump when the light is mechanically unblocked partially due to light leakage through the modulator. Whereas most transients are due to probe damage, some may result from multiple time constants. Figure 4(a) shows the IR signal while being damaged. The IR signal increases almost linearly with time (besides modulation effects). This is typical. Figure 4(b) illustrates probe damage with a complicated onset. Figure 4(c) illustrates a change in mechanism of 


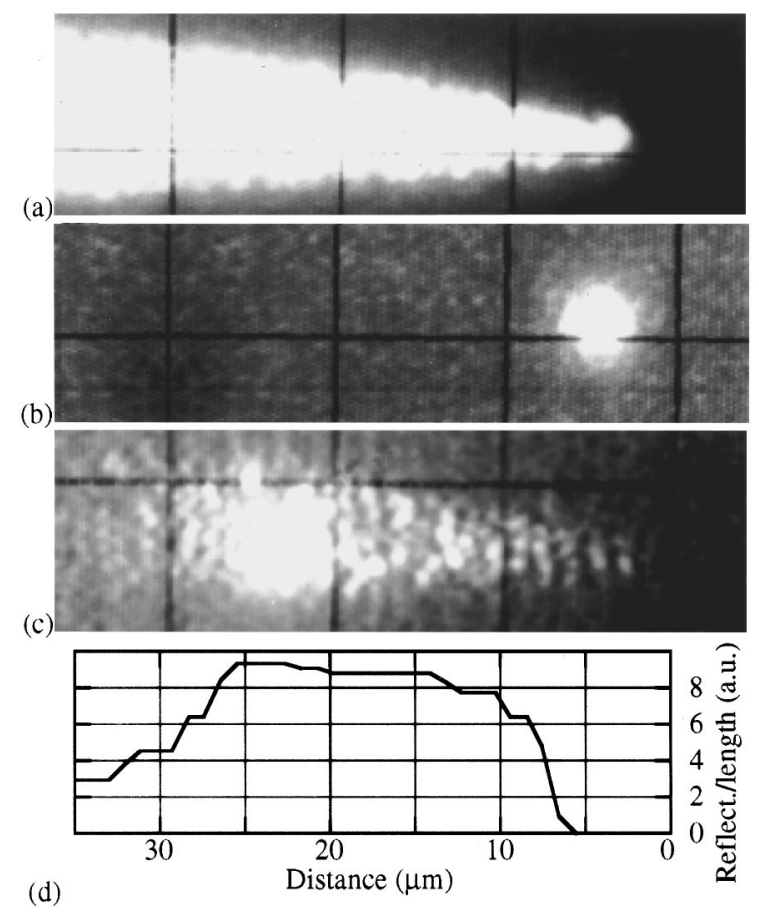

FIG. 3. Optical micrographs of a chubby probe illustrate a typical (a) probe shape as viewed by external illumination, (b) optical emission of the same new aluminum coated probe from a point at the end, and (c) the optical emission of the same probe after damage by coupling $14 \mathrm{~mW}$ of green light into the probe. The scale of the dark grids in the micrographs is $10 \mu \mathrm{m}$ per box. For comparison, the results of a ray tracing model described in the text which counts the number of ray bounces per length is shown in (d).

modulation: it begins positively correlated $(+)$ with the visible and ends negatively correlated $(-)$. One can explain such a sign change. If thermal elongation (-) initially dominates, damage can move the effective aperture back from the tip making it less important until aperture size $(+)$ dominates. Further damage could allow another sign change (observed) as the reflectance $(-)$ becomes dominate. Note that

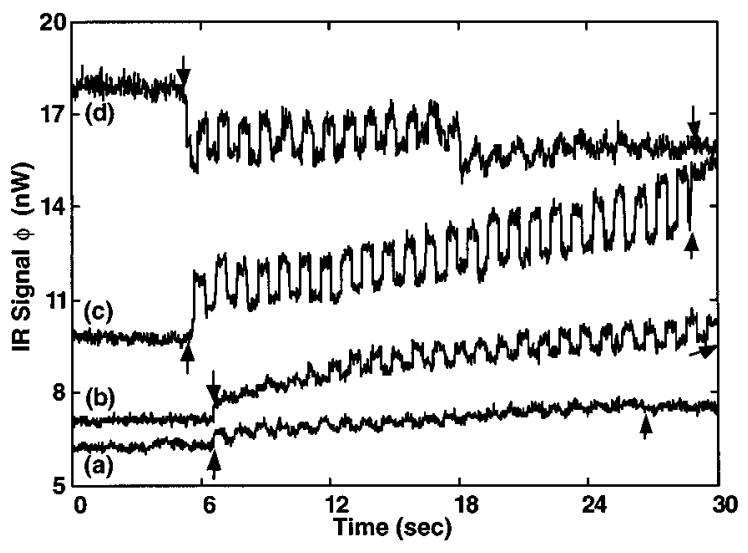

FIG. 4. The IR signal $\phi+\Delta \phi$ transmitted through the probe is recorded as a function of time for no visible light (before first arrow), while visible light is switched on and off at $1 \mathrm{~Hz}$, and after the visible light is again blocked (after second arrow). In (b), the second arrow occurred after the data shown, at which time the IR signal returned to the lower level, $\sim 9.7 \mathrm{nW}$. All used 1 $\mathrm{mW}$ of $\mathrm{cw}$ IR input to the probe, and maximal green light powers of (a) 3 $\mathrm{mW}$, (b) $4.5 \mathrm{~mW}$, (c) $7 \mathrm{~mW}$, and (d) $7 \mathrm{~mW}$. The probe was chubby with a $100 \mathrm{~nm}$ thick aluminum coating. correlation refers to the change as the visible light is switched and not the average value of the IR signal. Figure 4(d) shows damage to a probe at high power levels.

We have performed additional experiments to verify the general nature of the phenomena: (a) When the probe is immersed in water, the heat effect decreases, $(\Delta \phi / \phi)_{\text {water }}$ $=0.09(\Delta \phi / \phi)_{\text {air }}$, as expected due to the additional channel for dissipation. (b) When a probe is brought towards a sapphire surface, the IR variation decreases, suggesting that a sample acts as a heat sink. The magnitude ${ }^{15}$ of the effect indicates that the temperature change is reduced by a factor of 3. Further measurements will allow a determination of the thermal flux into the sample.

In conclusion, we have identified the mechanisms by which the heating of a NSOM probe affects the transmission of light through it. At high input power levels, damage to the probe was observed in real time. We have presented a systematic study of the coupling of thermal energy into the probe by identifying the location of maximal thermal input, which has been corroborated by several measurements and modeling. It is found to be $\sim 25 \mu \mathrm{m}$ from the tip, where the taper is large enough to allow manipulation of the probe properties. The thermal time constant of the probes was remarkably independent of probe geometry. It was found to be rather slow, $\sim 10 \mathrm{~ms}$, suggesting that a large fraction of the taper is heated above ambient. The slow response allows thermal effects to be distinguished from higher rate processes in the sample. ${ }^{16,17}$ This work was supported by the Army Research Office Grants DAAH04-94-G-0156 and DAAH0493-G-0194.

${ }^{1}$ E. Betzig and J. K. Trautman, Science 257, 189 (1992).

${ }^{2}$ D. W. Pohl, W. Denk, and M. Lanz, Appl. Phys. Lett. 44, 651 (1984).

${ }^{3}$ S. Xie and R. C. Dunn, Science 265, 361 (1994).

${ }^{4}$ H. J. Mamin and D. Rugar, Appl. Phys. Lett. 61, 1003 (1992).

${ }^{5}$ E. Betzig, J. K. Trautman, J. S. Weiner, T. D. Harris, and R. Wolfe, Appl. Opt. 31, 4563 (1992).

${ }^{6}$ Sutter Instruments Model P-2000, see, K. T. Brown and D. G. Flaming, Advanced Micropipette Techniques for Cell Physiology (Wiley, Chichester, 1986).

${ }^{7}$ B. I. Yakobson, P. J. Moyer, and M. A. Paesler, J. Appl. Phys. 73, 7984 (1993).

${ }^{8}$ H. A. Bethe, Phys. Rev. 66, 163 (1944).

${ }^{9}$ G. A. Massey, Appl. Opt. , 658 (1984).

${ }^{10}$ E. A. Ash and G. Nicholls, Nature 237, 510 (1972.

${ }^{11}$ L. Novotny and D. W. Pohl, Proceedings of NATO ASI Series E on Photons and Local Probes (Kluwer, Dordrecht, 1994).

${ }^{12} \mathrm{~J}$. M. Ziman, Principles of the Theory of Solids (University Press, Cambridge, 1969).

${ }^{13}$ H. S. Carslaw and J. C. Jaeger, Conduction of Heat in Solids (Clarendon, Oxford, 1960).

${ }^{14}$ B. I. Yakobson and M. A. Paesler, Ultramicroscopy 57, 204 (1995).

${ }^{15}$ A. H. LaRosa, B. I. Yakobson, M. A. Paesler, and H. D. Hallen, Proceedings of Conference on Near Field Optics and Related Techniques NFO-3, Brno, Czech Republic, Ultramicroscopy (1995).

${ }^{16}$ A. H. LaRosa, C. L. Jahncke, and H. D. Hallen, SPIE Proc. 2384, 101 (1995).

${ }^{17}$ A. LaRosa, C. L. Jahncke, and H. D. Hallen, Ultramicroscopy 57, 303 (1995). 